\title{
Survival Analysis of Patients with Breast Cancer Undergoing a Modified Radical Mastectomy With or Without a Thoracic Paravertebral Block: a 5-Year Follow-up of a Randomized Controlled Trial
}

\author{
MANOJ KUMAR KARMAKAR ${ }^{1}$, WINNIE SAMY ${ }^{1}$, ANNA LEE ${ }^{1}$, JIA WEI LI ${ }^{1}$, \\ WING CHEONG CHAN ${ }^{2}$, PHOON PING CHEN ${ }^{3}$ and BAN C.H. TSUI ${ }^{4}$ \\ ${ }^{1}$ Department of Anesthesia and Intensive Care, The Chinese University of Hong Kong, \\ Prince of Wales Hospital, Shatin, Hong Kong, P.R. China; \\ ${ }^{2}$ Department of Surgery, North District Hospital, Sheung Shui, New Territories, Hong Kong, P.R. China; \\ ${ }^{3}$ Departments of Anesthesiology and Operating Services, North District Hospital, \\ Sheung Shui, New Territories, Hong Kong, P.R. China; \\ ${ }^{4}$ Department of Anesthesiology, Perioperative and Pain Medicine, \\ Stanford University School of Medicine, Stanford, CA, U.S.A.
}

\begin{abstract}
Aim: This 5-year prospective follow-up of women randomized to general anesthesia (GA) with or without a thoracic paravertebral block (TPVB) examined the risk of local recurrence, metastasis and mortality after breast cancer surgery. Patients and Methods: A total of 180 patients undergoing modified radical mastectomy were randomized to one of three study groups: standardized GA only; GA with a single-injection TPVB ( $s-T P V B)$ and placebo paravertebral infusion after surgery for 72-h; and GA plus with continuous TPVB (c-TPVB) for 72- $h$ postoperatively. Cox proportional models were used to assess the effect of TPVB on long-term outcomes. Equivalence testing was used to help interpret the results. Results: The incidence [95\% confidence interval (CI)] of cancer recurrence, metastatic spread and all-cause mortality was $2.3 \%$ (0.7-5.4\%), 7.9\% (4.6-12.6\%) and $6.8 \%$ (3.6-11.2\%), respectively. Four women had cancer recurrence and had metastatic spread. Compared to the GA-only group, the risk of metastatic spread was not different from that of $G A$ with $s-T P V B$ [hazard ratio $(H R)=1.11,95 \% C I=0.32-3.83$ )
\end{abstract}

Correspondence to: Ban C. H. Tsui, Dip Eng, BSc (Math), BSc (Pharm), MSc (Pharm), MD, FRCP(C), PG Dip. Echo, Professor of Anesthesia, Department of Anesthesiology, Perioperative and Pain Medicine, Stanford University School of Medicine, 300 Pasteur Drive, H3582, Stanford, CA 94305-5640, U.S.A. Tel: +1 6507236412, Fax:+ 16507258544 , e-mail: bantsui@stanford.edu

Key Words: Breast cancer, radical mastectomy, paravertebral block, randomized controlled trial, survival. nor from that with GA plus c-TPVB $(H R=0.79,95 \% C I=0.21$ 2.96) ( $p=0.88)$. Compared to the GA-only group, the risk of mortality was similarly not different from that of the two other groups $(H R=2.57,95 \% C I=0.66-9.92$; and $H R=0.66,95 \%$ $C I=0.11-3.97$, respectively, $p=0.15)$. Conclusion: Although the original study was underpowered to properly address long-term outcomes, the results of this analysis suggest that $T P V B$, administered whether as a single-injection or continuous infusion during the perioperative period, had little to no appreciable effect on local recurrence, metastasis or mortality after breast cancer surgery.

Surgery offers the best prognosis for all excisable tumors. However, all general anesthetics (GAs) affect immune responses by depressing bone marrow activity, altering phagocytosis by macrophages, and inducing immunosuppression during surgery and in the postoperative period (1). Such immune compromise could affect the postoperative infection rate, healing, and rate and extent of tumor dissemination during surgery (2). Anesthesia and surgical procedures have also been reported to suppress natural killer cell activity and other immune functions for a few days after surgery (2). Surgery-induced immunosuppression is also associated with increased risk of tumor development and metastasis in laboratory studies $(2,3)$.

Regional anesthesia and analgesia has been suggested to have a potentially important role in preserving immune function by attenuating or preventing the immunosuppressive effect of surgery (2). A retrospective study suggested that patients given regional anesthesia for breast cancer have 
better prognosis than those given GA alone (4). When regional anesthesia and GA are combined, the amount of GA required is significantly reduced, as is, presumably, immune suppression. Furthermore, regional analgesia provides excellent pain relief, essentially reducing or obviating the need for postoperative opioids and consequent adverse effects on immune function and promotion of tumor growth (5). Regional analgesia also reduces the release of endogenous opioids (6), which exert a negative effect on the immune system. Certain GA regimens have been suggested to have potential in influencing cancer recurrence and metastasis; for example, a propofol thoracic paravertebral block (TPVB) technique was found to significantly reduce the concentrations of endogenous factors associated with angiogenesis in breast cancer (7).

We previously examined chronic pain and quality of life outcomes in a cohort of patients who underwent modified radical mastectomy for breast cancer who were randomized to receive a standardized GA regimen with or without a TPVB (8). In the current investigation, we prospectively assessed the same cohort for local cancer recurrence, metastasis and mortality for the 5-year period after surgery.

\section{Patients and Methods}

This trial was registered with the Centre for Clinical Trials of The Chinese University of Hong Kong, http://www.cct.cuhk.edu.hk/ Registry/publictriallist.aspx, under the unique trial registration number CUHK_CCT00301. Research Ethics Committee approval was obtained (CRE-2011.286) from the Joint Chinese University of Hong Kong-New Territories East Cluster Clinical Research Ethics Committee to conduct the 5-year follow up. Since there was no direct patient contact during the 5-year follow-up period informed consent was waived.

Population and sample size. This study was a follow-up study to a prospective, randomized, double-blinded controlled study on 180 women undergoing modified radical mastectomy with TPVB and GA (8). Details of the inclusion and exclusion criteria, randomization procedure, anesthetic technique and the paravertebral infusion regimen (72-h postoperatively) used are presented in detail in our previous report (8).

Briefly, adult patients American Society of Anesthesiologists (ASA) 1-3 under the age of 70 years undergoing major breast cancer surgery (which included axillary lymph node dissection) were recruited for this study. Patients with following conditions were excluded from the study: history of chronic pain, history of regular analgesic usage, contraindication to the use of non-steroidal antiinflammatory drugs, known allergy to local anesthetic drugs, infection at the site of block placement, bleeding tendency or with evidence of coagulopathy, pre-existing neurological or muscular disorders, history of psychiatric illness, pregnant patients, preexisting nausea or vomiting, patients who in the $24 \mathrm{~h}$ before surgery received opioid or drugs with antiemetic properties, patients who received chemotherapy or radiotherapy before surgery and patients with breast pain before surgery (8). For the long-term outcomes, the study data were collected prospectively from the same group of patients and from the Clinical Management System database, a computer database of the Hong Kong Hospital Authority, for the 5year period after surgery.

The study randomized 180 women into three groups (Figure 1) as follows: control: standardized general anesthesia (GA) using total intravenous anesthesia with propofol; sTPVB: GA with singleinjection TPVB (ropivacaine $2 \mathrm{mg} / \mathrm{kg}$ with $5 \mu \mathrm{g} / \mathrm{ml}$ epinephrine) and a paravertebral infusion of $0.9 \%$ saline $(0.1 \mathrm{ml} / \mathrm{kg} / \mathrm{h})$; and c-TPVB: general anesthesia with a single-injection TPVB as above and continuous paravertebral infusion of $0.25 \%$ ropivacaine at $0.1 \mathrm{ml} / \mathrm{kg} / \mathrm{h}$ for $72 \mathrm{~h}(8)$.

Outcome measures. The primary outcome measure was the incidence of local cancer recurrence or metastatic spread among the three groups. There was no direct patient contact or intervention during the follow-up. Data on the tumor, including tumor location, type, size, stage, and histological grading; resection margin; estrogen receptor status; progesterone receptor status; expression of human epidermal growth factor receptor (HER2); any lymphoangioinvasive growth; nodal status; adjuvant therapy received; immediate postoperative wound complications and treatment; and complications after receiving chemotherapy/ radiotherapy; and the data of recurrence or metastatic spread were recorded from the Clinical Management System database (4, 9). Mammograms, ultrasound, biopsy results, and follow-up records were also retrieved from the Clinical Management System database to review patient's progress and confirm any recurrence or metastasis. If there was any uncertainty on the patient's progress, consultation with the surgeons was initiated.

Statistical analysis. Data were analyzed using SPSS for windows (Version 22; IBM Corp, Armonk, NY, USA) and STATA 13.1 (StataCorp, College Station, TX, USA). Normality of the data was tested using Shapiro-Wilk's test. Data are presented as median and interquartile range (IQR) or frequency (\%). Kruskal-Wallis test was used to compare the medians between the study groups. The chisquare test was used to compare categorical data between groups.

The time from treatment allocation to first recurrence of breast cancer; metastasis to bone, liver, brain, or lung; or end of followup was recorded in months for the primary outcome. Similarly, time from treatment allocation to all-cause mortality or end of follow-up was estimated for the secondary outcome. The incidences of primary and secondary outcomes were estimated with $95 \%$ confidence intervals $(95 \% \mathrm{CI})$. As the follow-up durations were different between groups, we also report the incidence rate (per 10,000 months) and 95\% CI. Cox regression was used to estimate the hazard ratio (HR) for comparing the outcomes between groups after checking the proportional hazards assumption with scaled Schoenfeld residuals. The Harrell's c-statistic was estimated to assess the overall discrimination of the survival analysis model.

As this is a secondary analysis of patients recruited into a randomized controlled trial, we did not perform a sample size calculation. As this study was not properly powered for long-term cancer outcomes, equivalence testing was performed instead of posthoc power analysis $(10,11)$. Using WinPEPI software 11.65 (http://software-nanono.blogspot.com/2011/10/winpepi-statisticalsoftware-for.html), we calculated and interpreted the $90 \%$ CIs using equivalence test to provide an estimate of the maximum effect size from regional blocks that was supported by the data at the $5 \%$ significance level $(10,11)$. 


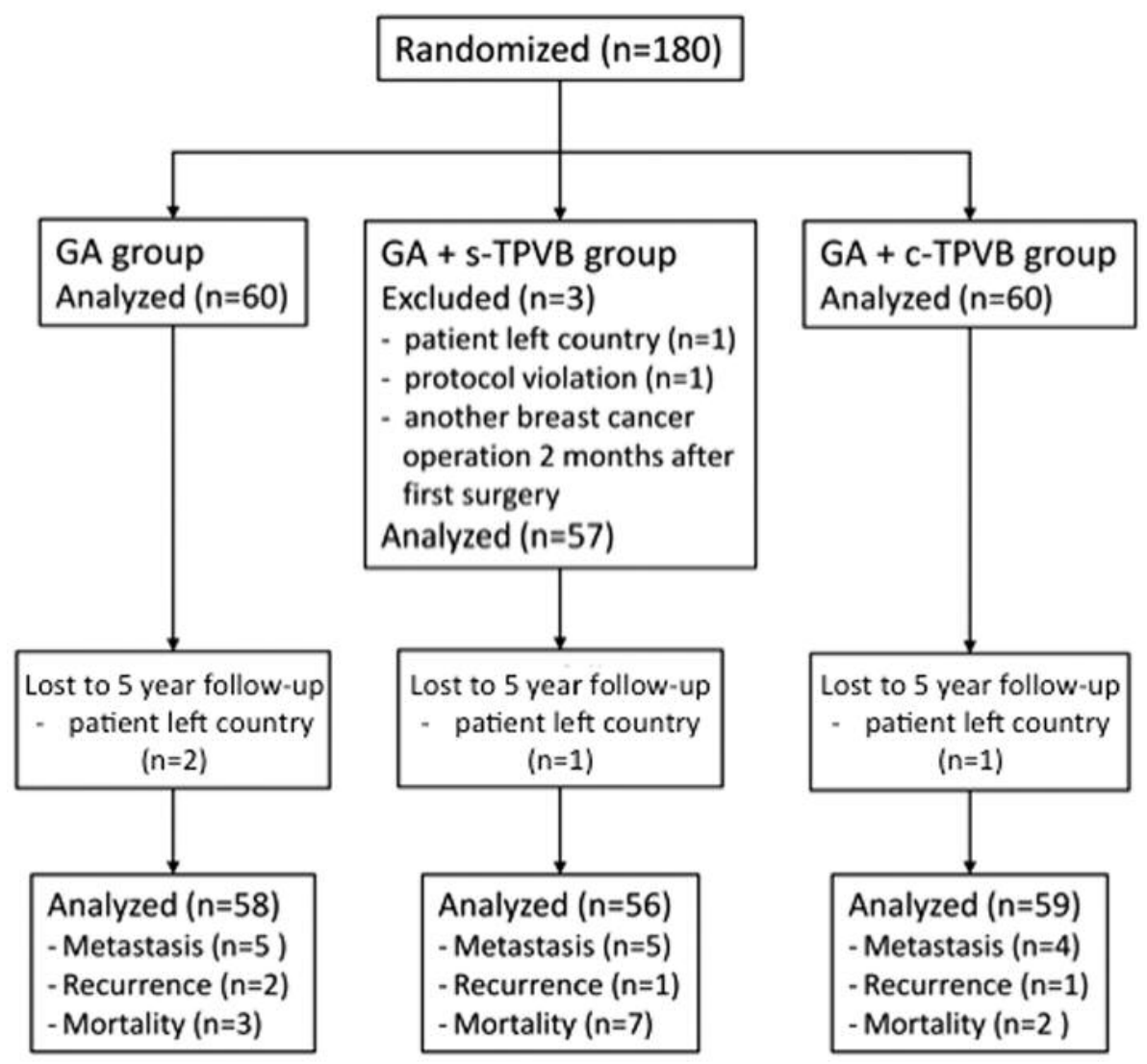

Figure 1. Patient flow chart. GA: General anesthesia; GA + s-TPVB: general anesthesia plus single-shot thoracic paravertebral block with placebo infusion; GA + c-TPVB: general anesthesia plus continuous paravertebral block infusion.

\section{Results}

The characteristics of the randomized patients in the three study groups were similar (Table I). As reported previously (8), the intraoperative median supplementary morphine requirement in the control group $[1.5(\mathrm{IQR}=0-3) \mathrm{mg}]$ was greater than that in the s-TPVB [0 (IQR=0-1) $\mathrm{mg}](p<0.001)$ and c-TPVB $[0(\mathrm{IQR}=0-1) \mathrm{mg}](p=0.001)$ groups. For the first $72 \mathrm{~h}$ after surgery, only one patient in the control group needed a single dose of intramuscularly administered morphine for rescue analgesia in the ward. Otherwise, there were no significant differences in resting or movement acute pain scores between the study groups.

Four women had local recurrence and metastasis occurred in all four. The incidences of local cancer recurrence, metastasis and all-cause mortality were $2.3 \%(95 \% \mathrm{CI}=0.7-$ $5.4 \%), 7.9 \%(95 \% \mathrm{CI}=4.6-12.6 \%)$ and $6.8 \%(95 \% \mathrm{CI}=3.6-$ $11.2 \%)$, respectively. There was no difference in the risk of local cancer recurrence, metastasis or all-cause mortality between the groups $(p=0.79, p=0.91$ and $p=0.13$, respectively). The incidence rate per 10,000 months of follow-up by study group are reported in Table II.

The $90 \% \mathrm{CI}$ of the difference in incidence of local recurrence between patients in the GA and GA plus s-TPVB and GA plus c-TPVB group were $1.6 \%(-4.9 \%$ to $8.1 \%)$ and $1.7 \%(-4.7 \%$ to $8.0 \%)$, respectively. For the difference in incidence of metastasis, the corresponding $90 \%$ CIs were $-0.4 \%(-10.7 \%$ to $9.8 \%)$ and $1.7 \%(-7.9 \%$ to $11.2 \%)$, respectively. For mortality, the corresponding $90 \%$ CIs were $-7.3 \%(-17.5 \%$ to $2.9 \%)$ and $1.7 \%$ ( $-6.0 \%$ to $9.3 \%)$, respectively. Neither local recurrence nor metastasis or mortality differed statistically with or without regional block. Using the equivalence testing method, we can reject the hypothesis that regional block with s-TPVB or c-TPVB makes a difference of greater than $10 \%$ compared to GA alone in local recurrence incidence at the 5\% significance level. Likewise, we can reject the hypothesis that the regional block with s-TPVB or c-TPVB makes a difference of greater than $15 \%$ compared with GA alone at the $5 \%$ significance level for metastasis incidence. Finally, for 
Table I. Characteristics of the breast tumors according to randomization to general anesthesia (GA) alone or with single (s-) or continuous (c-) thoracic paravertebral block (TPVB). Data are presented as frequency, $n(\%)$, or as median (interquartile range).

\begin{tabular}{|c|c|c|c|c|}
\hline & GA $(n=60)$ & $\mathrm{GA}+\mathrm{s}-\mathrm{TPVB}(\mathrm{n}=57)$ & $\mathrm{GA}+\mathrm{c}-\mathrm{TPVB}(\mathrm{n}=60)$ & $p$-Value \\
\hline Age (years) & $51(45-57)$ & $52(48-61)$ & $51(48-59)$ & 0.12 \\
\hline \multicolumn{5}{|l|}{ TNM Tumor staging $(\mathrm{n}, \%)$} \\
\hline $\mathrm{T} 1$ & $25(42)$ & $21(37)$ & $29(48)$ & \multirow{4}{*}{0.21} \\
\hline $\mathrm{T} 2$ & $31(52)$ & $34(59)$ & $25(42)$ & \\
\hline $\mathrm{T} 3$ & $2(3)$ & $2(4)$ & $1(2)$ & \\
\hline NA & $2(3)$ & $0(0)$ & $5(8)$ & \\
\hline \multicolumn{5}{|l|}{ TNM Lymph node staging $(\mathrm{n}, \%)$} \\
\hline No & $33(55)$ & $34(60)$ & $34(57)$ & \multirow{5}{*}{0.19} \\
\hline $\mathrm{N} 1$ & $15(25)$ & $15(26)$ & $10(17)$ & \\
\hline $\mathrm{N} 2$ & $8(13)$ & $4(7)$ & $10(17)$ & \\
\hline N3 & $2(3)$ & $4(7)$ & $1(2)$ & \\
\hline NA & $2(3)$ & $0(0)$ & $5(8)$ & \\
\hline \multicolumn{5}{|l|}{ TNM Distant metastasis staging $(\mathrm{n}, \%)$} \\
\hline M0 & $58(97)$ & $55(96)$ & $55(92)$ & \multirow[t]{3}{*}{0.05} \\
\hline M1 & $0(0)$ & $2(4)$ & $0(0)$ & \\
\hline NA & $2(3)$ & $0(0)$ & $5(8)$ & \\
\hline \multicolumn{5}{|l|}{ Histological grading $(\mathrm{n}, \%)^{*}$} \\
\hline Grade I & $16(27)$ & $14(25)$ & $15(25)$ & \multirow{4}{*}{0.67} \\
\hline Grade II & $23(38)$ & $24(42)$ & $29(49)$ & \\
\hline Grade III & $18(30)$ & $17(30)$ & $11(18)$ & \\
\hline NA & $3(5)$ & $2(3)$ & $5(8)$ & \\
\hline Tumor size at histology $(\mathrm{cm})$ & $2.3(1.3-3.0)$ & $2.5(1.6-3.2)$ & $2.1(1.5-3.2)$ & 0.74 \\
\hline NPI & $4.3(3.5-5.0)$ & $4.3(3.4-4.7)$ & $4.3(3.3-4.9)$ & 0.98 \\
\hline Lymphovascular invasion-positive (n,\%) & $22(36)$ & $21(37)$ & $24(40)$ & 0.33 \\
\hline ER-positive $(\mathrm{n}, \%)$ & $42(70)$ & $41(72)$ & $39(65)$ & 0.30 \\
\hline PR-positive $(\mathrm{n}, \%)$ & $36(60)$ & $40(70)$ & $33(55)$ & 0.13 \\
\hline HER2-positive (n,\%) & $3762)$ & $34(59)$ & $30(50)$ & 0.25 \\
\hline Chemotherapy at 3 months $(\mathrm{n}, \%)$ & $46(77)$ & $41(72)$ & $36(60)$ & 0.13 \\
\hline Radiotherapy at 3 months $(\mathrm{n}, \%)$ & $3(5)$ & $4(7)$ & $5(8)$ & 0.77 \\
\hline Chemotherapy at 6 months $(\mathrm{n}, \%)$ & $46(77)$ & $41(72)$ & $35(58)$ & 0.08 \\
\hline Radiotherapy at 6 months $(\mathrm{n}, \%)$ & $15(25)$ & $14(25)$ & $12(20)$ & 0.77 \\
\hline Received chemotherapy, radiation and endocrine therapy $(\mathrm{n}, \%)$ & $23(38)$ & $19(33)$ & $22(37)$ & 0.85 \\
\hline
\end{tabular}

TNM, Tumor node metastasis; NA, not applicable; DCIS, ductal carcinoma in situ; HER, human epidermal growth factor receptor; NPI: Nottingham Prognostic Index $=0.2 \times$ tumor size + histological grade + lymph node status. *Bloom-Richardson.

Table II. Incidence rate [95\% confidence interval (CI)] of local recurrence, metastasis, and mortality after the breast cancer surgery and location of metastasis by group.

\begin{tabular}{lccc}
\hline & GA $(\mathrm{n}=60)$ & $\mathrm{GA}+\mathrm{s}-\mathrm{TPVB}(\mathrm{n}=57)$ & $\mathrm{GA}+\mathrm{c}-\mathrm{TPVB}(\mathrm{n}=60)$ \\
\hline Local recurrence (per 10,000 months) & $5.4(1.3-21.4)$ & $3.1(0.4-22.0)$ & $2.8(0.4-19.9)$ \\
Metastasis (per 10,000 months) & $13.4(5.6-32.2)$ & $15.5(6.4-37.2)$ & $11.2(4.2-29.8)$ \\
Site of metastasis, $\mathrm{n}$ & & & 1 \\
$\quad$ Bone & 0 & 0 & 0 \\
Liver & 1 & 1 & 0 \\
Brain & 0 & 2 & 1 \\
Lung & 1 & 1 & 0 \\
Bone +brain & 1 & 0 & 2 \\
Bone + lung & 1 & 0 & 0 \\
Bone + liver & 0 & 1 & 0 \\
Bone + lung + liver & 1 & 0 & \\
All-cause mortality (per 10,000 months) & $7.9(2.5-24.4)$ & $21.1(10.1-44.3)$ & $5.5(1.4-22.0)$ \\
\hline
\end{tabular}

GA: General anesthesia; GA + s-TPVB: general anesthesia plus single-shot thoracic paravertebral block with placebo infusion; GA + CTPVB: general anesthesia plus continuous paravertebral block infusion. 


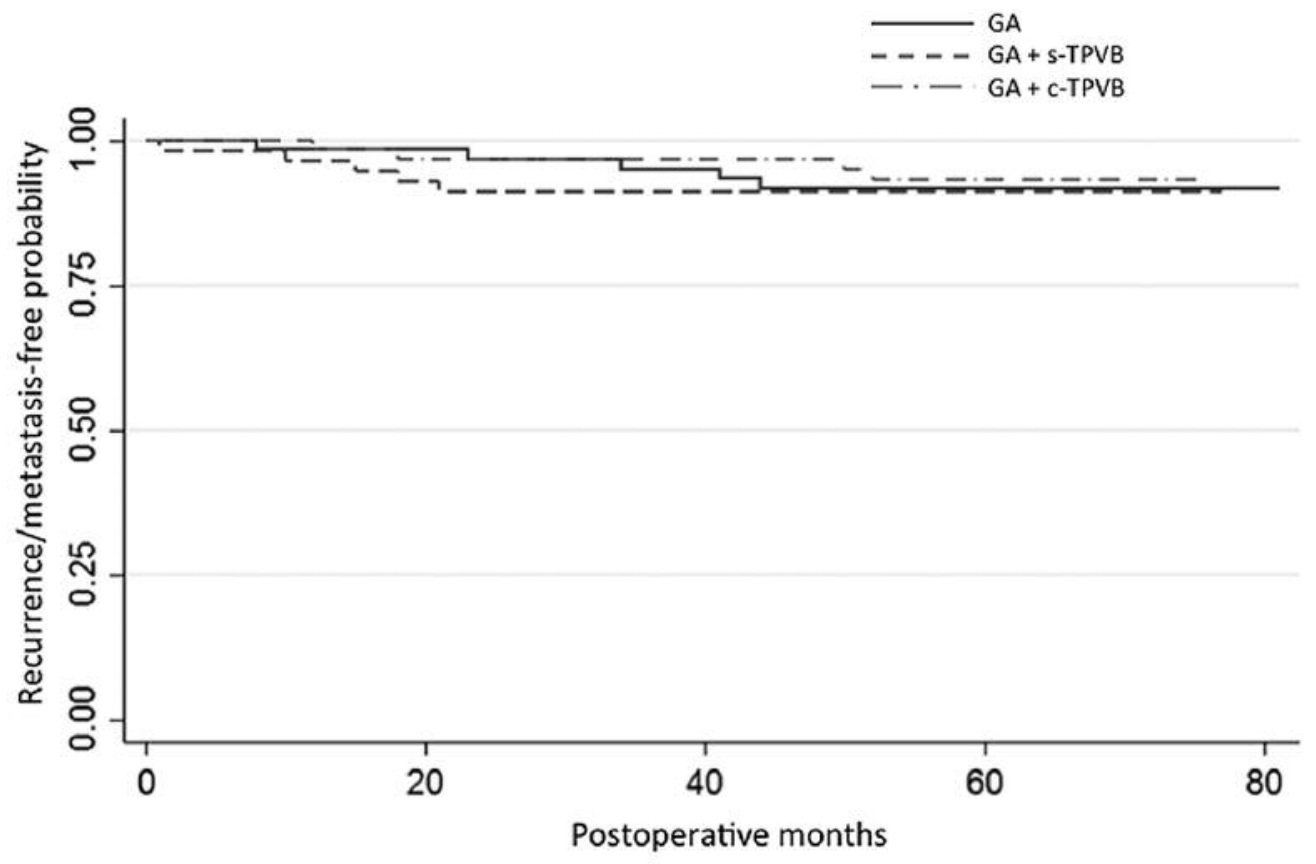

Figure 2. Local recurrence or metastasis-free probability estimates by group. GA: General anesthesia; GA + s-TPVB: general anesthesia plus singleshot thoracic paravertebral block with placebo infusion; $G A+c-T P V B$ : general anesthesia plus continuous paravertebral block infusion ( $p=0.88$ ).

mortality, we can reject the hypothesis that the regional block with s-TPVB or c-TPVB makes a difference in incidence of greater than $20 \%$ compared to GA alone at the $5 \%$ significance level.

With regard to the primary outcome, the median duration of follow-up was 60 months (range=1-81 months), with a total follow-up time of 10,540 months. The proportional hazards assumption was met $(p=0.13)$. There was no difference in the risk of local cancer recurrence or metastasis between groups ( $p=0.88$, Figure 2). Compared to the GAonly group, the risk of local recurrence or metastasis was no different to that for patients in the GA plus s-TPVB group $(\mathrm{HR}=1.11,95 \% \mathrm{CI}=0.32-3.83)$ and in the GA plus c-TPVB group $(\mathrm{HR}=0.79,95 \% \mathrm{CI}=0.21-2.96)$. The discrimination of the model was poor $(\mathrm{c}-$ statistic $=0.54)$.

The median duration of follow-up for all-cause mortality was 60 months (range $=11-81$ months), with a total followup time of 10,761 months. The proportional hazards assumption was met $(p=0.63)$. There was no difference in risk of mortality between groups ( $p=0.15$, Figure 3$)$. Compared to the GA-only group, the risk of death was no different to that of patients in the GA plus s-TPVB group $(\mathrm{HR}=2.57,95 \% \mathrm{CI}=0.66-9.92)$ and in the GA plus c-TPVB group $(\mathrm{HR}=0.66,95 \% \mathrm{CI}=0.11-3.97)$. The discrimination of the model was fair $(\mathrm{c}-$ statistic $=0.65)$. Survival probability estimates by group are summarized in Figure 3 .

\section{Discussion}

This is the first long-term follow-up to a randomized controlled trial examining the effects of TPVB (singleinjection or continuous infusion) given to women undergoing a modified radical mastectomy for breast cancer with a curative intent. This study was originally designed to evaluate the effects of a TPVB on both short-term outcomes (up to 6 months after surgery), i.e. the incidence of chronic pain and health-related quality of life, and long-term outcomes (up to 5 years after surgery), i.e. overall 5-year survival, after modified radical mastectomy. We previously reported that there was no significant difference in the incidence or relative risk of chronic pain at 3 and 6 months after modified radical mastectomy whether patients received a TPVB or not (8), but patients who received a TPVB reported less severe chronic pain, exhibited fewer symptoms and signs of chronic pain, and also experienceed a better physical and mental state (8). Now, 5 years after the study intervention, we have found no significant difference between the three study groups with regards to local cancer recurrence, metastasis or all-cause mortality. However, we acknowledge that the study was underpowered to rigorously test these long-term outcomes. Overall, our results suggest that TPVB offers some short-term benefits with regards to the intensity of chronic pain (verbal rating scale) and mental 


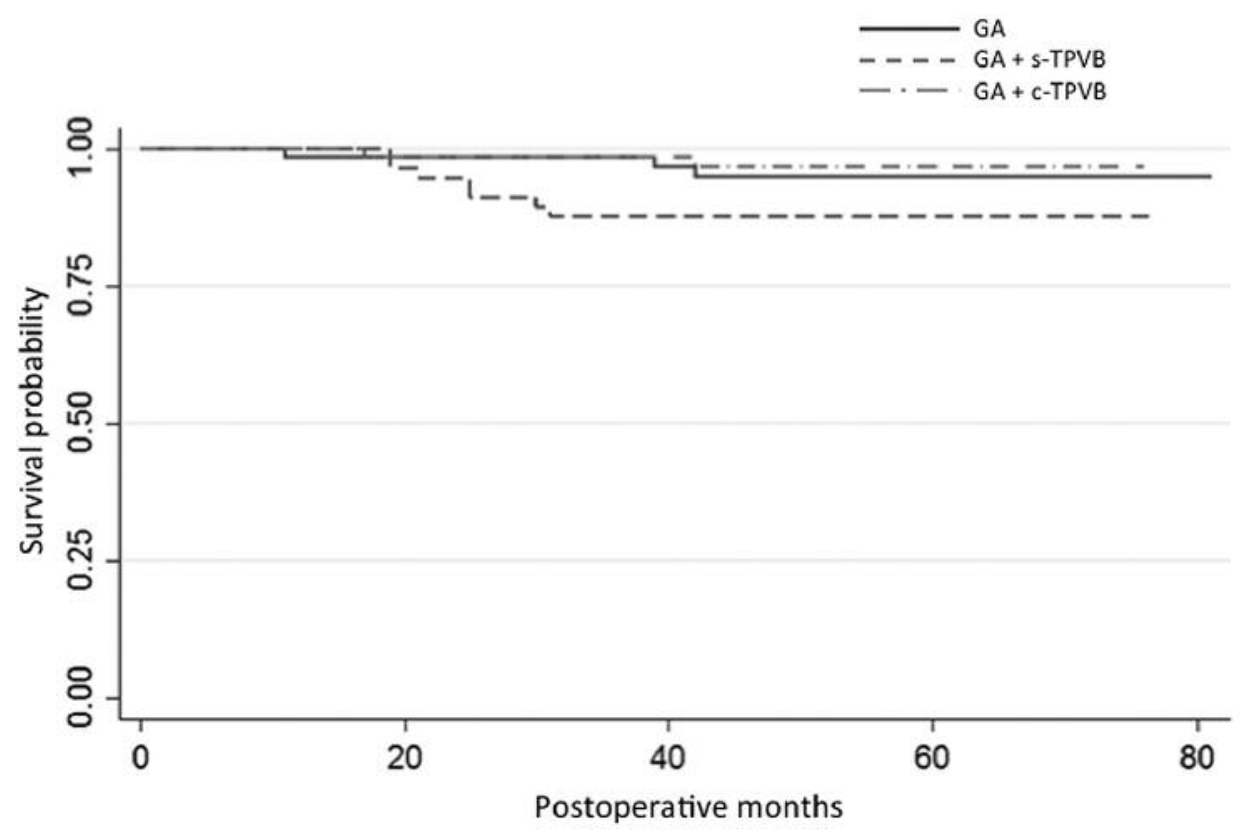

Figure 3. Survival probability estimates by group. GA: General anesthesia; GA + s-TPVB: general anesthesia plus single-shot thoracic paravertebral block with placebo infusion; $G A+c$-TPVB: general anesthesia plus continuous paravertebral block infusion ( $p=0.15)$.

health-related quality of life, but there is no clear evidence to support TPVB affecting any long-term outcomes related to disease and mortality after breast cancer surgery.

The premise behind using regional anesthesia for cancer surgery is immunoprotection; the immune system plays a critical role in attacking cancer cells and, when compromised by, for example, stress associated with surgery or pharmacological agents such as opioids, it is thought that there is a greater risk of recurrence or metastasis following surgery (12). While regional anesthesia is not expected to adversely affect the immune system (13), its addition to the anesthetic regimen does not seem to have any effect on longterm outcomes after modified radical mastectomy for breast cancer. Previous observational studies on breast surgery for cancer $(4,14)$ have yielded results suggesting that regional anesthesia has negligible, if any, effect on cancer recurrence and mortality/survival. Interestingly, there are limited data suggesting that the use of regional anesthesia for ovarian cancer surgery may significantly improve survival and recurrence outcomes $(15,16)$. It is possible that other forms of analgesia, such as non-steroidal anti-inflammatory drugs, may confer reduced recurrence rates following breast cancer surgery (17). Thus, further long-term prospective, highquality research into anesthetic and analgesic regimens for this class of surgery is warranted.

In this study, the 5-year overall survival and recurrence rates appear better than those predicted by the Nottingham Prognostic Index (NPI) $(18,19)$. For each of our groups, the median NPI score was 4.3 (Table I), with a survival rate of 93-98\%, which is within the NPI category 3 range with a recently reported survival rate of $90 \%$ (19). The higher survival rates among our groups could be explained by multiple factors. Long-term survival rates following breast cancer diagnosis stood at only $77 \%$ using the same index 30 years ago (18); in contrast, a recent study showed overall 5year survival rates had improved substantially to $90 \%$ for NPI category 3 cases (19). Increased use of improved screening methods and superior treatment regimens for breast cancer may account for overall improved survival rates of patients with breast cancer in recent decades. Alternatively, since all our patients received total intravenous anesthesia with propofol, regardless of whether TPVB was administered, it is possible that the components of the GA, and in particular propofol, conferred an unanticipated benefit. This is not unexpected, as propofol has numerous documented positive effects on immune system function (3, 20-24). Nonetheless, more research needs to be carried out to clarify the value of propofol and total intravenous anesthesia in mitigating cancer recurrence and increasing survival rates.

One of the strengths of the current study over previous studies, as well as some ongoing studies, is that total intravenous anesthesia was used for induction and maintenance of GA in all groups. For the GA-only groups of other studies, inhalational anesthetic agents, including sevoflurane and isoflurane, were used for induction and maintenance of GA. Current evidence suggests that inhaled 
anesthetics are, in general, immunosuppressive, whereas propofol has been shown to exert minimal negative effects on cell-mediated immunity (13). Indeed, a recent study comparing total intravenous anesthesia to inhaled volatile anesthetics revealed an association between propofol use and lower metastasis incidence (25). An ongoing multicenter study (Clinicals.gov NCT00418457) used total intravenous anesthesia for GA in the study group (also administering TPVB) (26); however, the combined use of sevoflurane and opioid in the control group makes it difficult to directly compare the true merits of regional anesthesia alone in the two groups with respect to the impact of anesthetic regimen on outcomes. Since all patients in our study received TIVA, this study should be able to examine the effects of the regional anesthetic technique on long-term outcomes in isolation by eliminating other possible confounding effects due either to the immunoprotective benefits of TIVA or the absence of immunoprotective effects of the TPVB.

It is important to note that the sample size of this study was determined and powered for the primary short-term outcome (namely the incidence of chronic pain) (8). Therefore, it is likely that the study was underpowered to detect any significant difference in long-term cancer outcomes or that no treatment effect exists. In fact, it has been suggested that over 1000 participants would be needed in a cancer prognosis study to provide $85 \%$ power to detect a $30 \%$ difference in effect (27), while an even larger population would obviously be needed to detect a smaller difference in effect. Thus, most studies of this nature require a substantial number of patients, which is only feasible with multicenter studies conducted over a number of years.

With an underpowered study such as ours, however, it is possible to estimate the maximum potential effect of regional anesthesia by applying the equivalence testing method utilizing the data from the study. Despite one can argue that the $90 \%$ CI was imprecise, the information generated from the equivalence test using the $90 \%$ CI can still be helpful when interpreting a negative study, particularly when considering the potential risk and benefit of an invasive intervention or procedure. Using the equivalence test, this study estimated the maximum potential effect of regional blocks with single shot or continuous catheter was no more than $10 \%$ on local recurrence, $15 \%$ on metastasis and $20 \%$ on overall mortality rates. While a maximum potential of $10 \%, 15 \%$ or $20 \%$ effect from regional anesthesia over GA alone may seem clinically significant at first glance, it is important to point out that the study result provides no guarantee that a regional block would achieve maximum potential, or even have any influence on local recurrence, metastasis and overall mortality at all. This is because these potential effects were projected for a maximum effect and not minimum effect of a regional block.
Another limitation of this study was the possibility of performance bias being present as the outcome assessors were not blinded. We believe that the level of ascertainment bias was low as our patients were unlikely to be treated in the private health system where the Clinical Management System database is not used. Attrition bias was low in this study as follow-up was complete in over $98 \%$ of cases.

In conclusion, our results suggest that TPVB does not affect long-term outcomes related to disease and mortality after breast cancer surgery. Our findings are in agreement with those of others (28) who also found no clear evidence that regional anesthesia offers supporting benefits for local cancer recurrence, metastasis, or survival following cancer surgery. However, we acknowledge that our sample size was insufficient to detect a treatment effect. Nevertheless, we must not overlook the immediate and well-proven benefits of regional anesthesia during the perioperative period. By trying to attribute long-term outcomes to a peripheral nerve block that was administered many years ago, we may be failing to appreciate the primary reason(s) for administering regional anesthesia. (29)

\section{Funding}

This work was locally funded by the Department of Anesthesia and Intensive Care, The Chinese University of Hong Kong, Prince of Wales Hospital, Shatin, Hong Kong, China. Dr. Ban Tsui was supported by a Clinical Scholar Award from the Alberta Heritage Foundation for Medical Research (AHFMR), Canada.

\section{Acknowledgements}

Dr. Tsui was supported by a Clinical Scholar Award from the Alberta Heritage Foundation for Medical Research (AHFMR) and Dr. Tsui's research was also supported by the Canadian Anesthesia Research Foundation.

\section{References}

1 Menges P, Kessler W, Kloecker C, Feuerherd M, Gaubert S, Diedrich S, van der Linde J, Hegenbart A, Busemann A, Traeger T, Cziupka K, Heidecke C-D and Maier S: Surgical trauma and postoperative immune dysfunction. Eur Surg Res 48: 180-186, 2012.

2 Shakhar G and Ben-Eliyahu S: Potential prophylactic measures against postoperative immunosuppression: could they reduce recurrence rates in oncological patients? Ann Surg Oncol 10: 972-992, 2003.

3 Deegan CA, Murray D, Doran P, Ecimovic P, Moriarty DC and Buggy DJ: Effect of anaesthetic technique on oestrogen receptornegative breast cancer cell function in vitro. Br J Anaesth 103: 685-690, 2009.

4 Exadaktylos AK, Buggy DJ, Moriarty DC, Mascha E and Sessler DI: Can anesthetic technique for primary breast cancer surgery affect recurrence or metastasis? Anesthesiology 105: 660-664, 2006. 
5 Snyder GL and Greenberg S: Effect of anaesthetic technique and other perioperative factors on cancer recurrence. $\mathrm{Br} \mathrm{J}$ Anaesth 105: 106-115, 2010.

6 Chae BK, Lee HW, Sun K, Choi YH and Kim HM: The effect of combined epidural and light general anesthesia on stress hormones in open heart surgery patients. Surg Today 28: 727731,1998

7 Looney M, Doran P and Buggy DJ: Effect of anesthetic technique on serum vascular endothelial growth factor $\mathrm{C}$ and transforming growth factor $\beta$ in women undergoing anesthesia and surgery for breast cancer. Anesthesiology 113: 1118-1125, 2010.

8 Karmakar MK, Samy W, Li JW, Lee A, Chan WC, Chen PP and Ho AM-H: Thoracic paravertebral block and its effects on chronic pain and health-related quality of life after modified radical mastectomy. Reg Anesth Pain Med 39: 289-298, 2014.

9 Esteve R, Marquina-Aponte V and Ramírez-Maestre C: Postoperative pain in children: association between anxiety sensitivity, pain catastrophizing, and female caregivers' responses to children's pain. J Pain 15: 157-168.e1, 2014.

10 Hoenig JM and Heisey DM: The Abuse of Power. Am Stat 55: 19-24, 2001.

11 Quertemont E: How to statistically show the absence of an effect. Psychol Belg 51: 109, 2011.

12 Neeman E and Ben-Eliyahu S: Surgery and stress promote cancer metastasis: new outlooks on perioperative mediating mechanisms and immune involvement. Brain Behav Immun 30(Suppl): S32-40, 2013.

13 Green JS and Tsui BCH: Impact of anesthesia for cancer surgery: Continuing professional development. Can J Anaesth 60: 1248-1269, 2013.

14 Koonce SL, Mclaughlin SA, Eck DL, Porter S, Bagaria S, Clendenen SR and Robards CB: Breast cancer recurrence in patients receiving epidural and paravertebral anesthesia: a retrospective, case-control study. Middle East J Anaesthesiol 22: 567-571, 2014.

15 de Oliveira GS, Ahmad S, Schink JC, Singh DK, Fitzgerald PC and McCarthy RJ: Intraoperative neuraxial anesthesia but not postoperative neuraxial analgesia is associated with increased relapse-free survival in ovarian cancer patients after primary cytoreductive surgery. Reg Anesth Pain Med 36: 271-277, 2011.

16 Lin L, Liu C, Tan H, Ouyang H, Zhang Y and Zeng W: Anaesthetic technique may affect prognosis for ovarian serous adenocarcinoma: a retrospective analysis. $\mathrm{Br} \mathrm{J}$ Anaesth 106: 814-822, 2011.

17 Forget P, Bentin C, Machiels J-P, Berliere M, Coulie PG and De Kock M: Intraoperative use of ketorolac or diclofenac is associated with improved disease-free survival and overall survival in conservative breast cancer surgery. Br J Anaesth 113: i82-i87, 2014.

18 Haybittle JL, Blamey RW, Elston CW, Johnson J, Doyle PJ, Campbell FC, Nicholson RI and Griffiths K: A prognostic index in primary breast cancer. Br J Cancer 45: 361-366, 1982.
19 Fong Y, Evans J, Brook D, Kenkre J, Jarvis P and GowerThomas K: The Nottingham Prognostic Index: five- and ten-year data for all-cause survival within a screened population. Ann R Coll Surg Engl 97: 137-139, 2015.

20 Blamey RW, Ellis IO, Pinder SE, Lee AHS, Macmillan RD, Morgan DAL, Robertson JFR, Mitchell MJ, Ball GR, Haybittle JL and Elston CW: Survival of invasive breast cancer according to the Nottingham Prognostic Index in cases diagnosed in 19901999. Eur J Cancer 43: 1548-1555, 2007.

21 Inada T, Yamanouchi Y, Jomura S, Sakamoto S, Takahashi M, Kambara $\mathrm{T}$ and Shingu K: Effect of propofol and isoflurane anaesthesia on the immune response to surgery. Anaesthesia 59: 954-959, 2004.

22 Kushida A, Inada T and Shingu K: Enhancement of antitumor immunity after propofol treatment in mice. Immunopharmacol Immunotoxicol 29: 477-486, 2007.

23 Melamed R, Bar-Yosef S, Shakhar G, Shakhar K and BenEliyahu S: Suppression of natural killer cell activity and promotion of tumor metastasis by ketamine, thiopental, and halothane, but not by propofol: mediating mechanisms and prophylactic measures. Anesth Analg 97: 1331-1339, 2003.

24 Ren XF, Li WZ, Meng FY and Lin CF: Differential effects of propofol and isoflurane on the activation of T-helper cells in lung cancer patients. Anaesthesia 65: 478-482, 2010.

25 Wigmore TJ, Mohammed K and Jhanji S: Long-term survival for patients undergoing volatile versus iv anesthesia for cancer surgery: a retrospective analysis. Anesthesiology 124: 69-79, 2016.

26 Wu J, Buggy D, Fleischmann E, Parra-Sanchez I, Treschan T, Kurz A, Mascha EJ and Sessler DI: Thoracic paravertebral regional anesthesia improves analgesia after breast cancer surgery: a randomized controlled multicentre clinical trial. Can J Anaesth 62: 241-251, 2015.

27 Sessler DI, Ben-Eliyahu S, Mascha EJ, Parat M-O and Buggy DJ: Can regional analgesia reduce the risk of recurrence after breast cancer? Methodology of a multicenter randomized trial. Contemp Clin Trials 29: 517-526, 2008.

28 Tsui BCH, Rashiq S, Schopflocher D, Murtha A, Broemling S, Pillay $\mathrm{J}$ and Finucane BT: Epidural anesthesia and cancer recurrence rates after radical prostatectomy. Can J Anaesth 57: 107-112, 2010.

29 Tsui BCH: Regional anesthesia: business class pain management? Reg Anesth Pain Med 39: 265-266, 2014. 